\title{
The quality of patients' self-blood pressure measurements: a cross-sectional study
}

\author{
Katarzyna Nessler ${ }^{1 *}$, Anna Krztoń-Królewiecka', Anna Suska², Mitchell R. Mann², Michał B. Nessler ${ }^{3}$ and \\ Adam Windak ${ }^{1}$
}

\begin{abstract}
Background: The accurate and independent measurement of blood pressure (BP) by patients is essential for home BP monitoring (HBPM) and determining the quality of hypertension (HTN) control. This study aimed to evaluate the BP self-measurement techniques of hypertensive patients and their accuracy in accordance with established guidelines. We sought to identify the common errors that patients make and suggest improvements that can be implemented in the primary healthcare setting to increase the reliability of HBPM conducted by hypertensive patients.

Methods: One hundred patients diagnosed with HTN completed a questionnaire inquiring about their health and demographic data and BP monitoring practices. Patients were then observed and filmed while measuring their BP on their own devices in five primary healthcare centres in Kraków, Poland. The correctness of their techniques was assessed in accordance with the European Society of Hypertension guidelines on HBPM.

Results: Only 3\% of patients measured their BP without error; $60 \%$ made three or more errors. The most frequent error, made by $76 \%$ of subjects, was incorrect sphygmomanometer cuff placement (above or below heart level, or/ and the indicator mark was not aligned with the brachial artery). Regarding patients' previous instruction for the correct use of their devices, $36 \%$ of patients referred to their monitor's user manual, $22 \%$ did not receive any prior assistance, and only $29 \%$ were adequately counselled by physicians on how to measure their BP correctly.

Conclusions: Our findings suggest that primary healthcare physicians and their personnel often do not adequately instruct patients on how to measure their BP correctly. Therefore, healthcare systems must provide patients with more adequate training and reference materials on the best practices of BP monitoring.
\end{abstract}

Keywords: Home blood pressure monitoring, HBPM, Hypertension management

\section{Background}

Hypertension (HTN) is one of the principal risk factors for cardiovascular disease and is responsible for the deaths of approximately nine million people annually worldwide [1]. The global prevalence of HTN amongst adults is $30-45 \%$; in Poland, it is approximately $32 \%$ [2, 3].

\footnotetext{
*Correspondence: katarzynanessler@gmail.com

${ }^{1}$ Department of Family Medicine, Jagiellonian University Medical College, Bocheńska 4, 31-061 Kraków, Poland

Full list of author information is available at the end of the article
}

Reliable blood pressure (BP) measurements are critical to the effective diagnosis and treatment of HTN. Compared with those of office BP measurement (OBPM), BP values obtained via home $\mathrm{BP}$ monitoring (HBPM) are typically lower [4].

HBPM is the average of all BP readings performed with a semiautomatic, validated BP monitor for at least three days and preferably six to seven consecutive days. Readings should be taken in the mornings and evenings in a quiet room after five minutes of rest and in a seated position with the back and arm supported [5]. 
Two measurements, one to two minutes apart, should be taken each time [5].

HBPM has been shown to provide more reproducible data than OBPM [4]. Recent meta-analyses show that HBPM-based treatments are strongly recommended in the control of HTN [6, 7]. Furthermore, recent studies have indicated that BP measurements made by patients at home better predict cardiovascular morbidity and mortality [8]. BP self-monitoring has also been shown to have a beneficial effect on medication adherence and BP control $[9,10]$. However, it has been observed that even minor systematic errors in BP measurements may cause substantial variations in the proportion of patients being diagnosed with HTN $[11,12]$. Moreover, this relatively simple technique is prone to numerous patient-caused errors that may significantly distort measurement results.

This study aimed to answer the following questions:

1. Do patients measure their BP in accordance with the standards set up by the European Society of Hypertension (ESH) guidelines [13]?

2. What are the most common errors made by patients when measuring their BP?

3. What sources of information about correct BP measurement techniques do patients use?

4. Are there any associations between errors made by patients during self-measurement of their BP and their personal characteristics?

\section{Methods}

\section{Study design}

This cross-sectional study was conducted between July 2016 and May 2018. Participants were recruited from five primary healthcare centres in the Kraków area in southern Poland, which agreed to participate in the study. Medical students from Jagiellonian University Medical College in Kraków were trained to act as research fieldworkers. They checked the inclusion criteria of patients who agreed to participate, obtained their informed consent, asked them to fill in the study questionnaire, and filmed their BP self-measurement routines. Study participants brought their own BP gauges and used them while demonstrating their BP self-measurement techniques. This study was approved by the Jagiellonian University Bioethics Committee (122.6120.121.2015; 25 June 2015) and was conducted according to good clinical practice $(\mathrm{GCP})$ rules.

\section{Sampling and study participants}

The minimum patient sample size $(n)$ calculated with OpenEpi software was estimated to be 97 (detailed information in Additional file 1, Additional file 2). One hundred forty-seven patients were approached as potential subjects in the order in which they applied for a medical appointment for any reason. The study participants were 100 consecutive patients who fulfilled the inclusion criteria and agreed to take part in the study (response rate: 68\%). The inclusion criteria were: (1) age of 18 or older; (2) diagnosed HTN in accordance with ESH guidelines, defined as an office systolic BP $\geq 140$ and/ or diastolic $\mathrm{BP} \geq 90 \mathrm{mmHg}$ [13]; (3) declared regular BP monitoring at home; (4) a lack of current or past arrhythmias; and (5) a lack of comorbidities that could prevent effective communication (e.g., cognitive, visual, or hearing impairments, motor difficulties, inabilities to give informed consent). No restrictions were implemented to select for patients' level of BP monitoring training.

\section{Measurements}

The questionnaire had two parts and consisted of 27 questions (Additional file 1, Additional file 2). Part one collected basic patient demographic and medical history data, while part two collected information on patient knowledge of HBPM techniques.

Patients sat comfortably in a quiet environment for five minutes and were then asked to measure their BP using their sphygmomanometers in the same way that they would at home. Each patient completed two BP measurements one to two minutes apart. A third measurement was performed if the first two readings differed by $>10$ $\mathrm{mmHg}$. BP values were recorded as the average of the last two readings. Patients were filmed for all proceedings and were aware of their surveillance throughout. Five minutes after the final BP measurement made by each patient, a researcher conducted a BP measurement with an upper arm automatic sphygmomanometer.

Two independent observers reviewed the footage of each patient and assessed its accordance with the 2010 ESH guidelines for HBPM [13]. In the case of disagreements between the two observers, a third independent opinion was sought for arbitration.

\section{Statistical analysis}

Statistical analyses were performed with Statistica 13.3 software (TIBCO Inc.). To present the results, we used descriptive statistics. To investigate the associations between specific errors made by patients and their characteristics, the Chi-square and Mann-Whitney $U$ tests were performed for qualitative and quantitative variables, respectively. To analyse the associations between the number of errors made and patient characteristics, we used the Mann-Whitney U test, the Kruskal-Wallis test, and Spearman's rank correlation coefficient. Multiple linear regression analysis was used to measure the final associations between patient characteristics and the 
number of errors made, adjusted for covariates. An alpha value of $p=0.05$ was considered statistically significant.

\section{Results \\ Respondent characteristics}

One hundred patients with a mean age of 66.19 years (SD $=10.07$ years; range: $36-85$ years) with diagnosed HTN were recruited. The sample consisted mostly of female patients (61\%) and those from a city of over 50,000 inhabitants (69\%). Forty-one per cent had a 1st to 3rd level European Qualifications Framework (EQF) education, $34 \%$ had a 4 th to 6 th level education, and $25 \%$ had a 7th or 8th level completed [14]. The average time from HTN diagnosis to the present study was 12.5 years ( $\mathrm{SD}=8.24$ years; range: $1-32$ years). The mean patient body mass index (BMI) was $29.95 \mathrm{~kg} / \mathrm{m}^{2}(\mathrm{SD}=4.76 \mathrm{~kg} /$ $\mathrm{m}^{2}$; range: $19.37-42.25 \mathrm{~kg} / \mathrm{m}^{2}$ ). Sixty-three per cent of patients had a family history of HTN; $29 \%$ of patients had coexisting chronic diseases.

Most patients used an upper arm automatic sphygmomanometer (64\%), 11\% used aneroid devices, and upper arm semi-automatic and wrist gauges were used by $7 \%$ and $18 \%$ of patients, respectively.

\section{Sources of information about correct BP self-measurement} The main source of information on BP self-measurement techniques was the sphygmomanometer user manual (36\%). Of the 19 patients given information by a general practitioner, 18 received oral instructions (11 observed a live demonstration). Ten patients were instructed by their cardiologist. One in five patients did not receive any information on how to measure their BP properly.

\section{Accuracy of BP self-measurement}

Only 3\% of patients made no errors while recording their BP. Sixty per cent of patients made three or more errors; the most frequent one, made by more than three-quarters of the study participants, was incorrect cuff placement (above or below heart level and/or the indicator mark was not aligned with the brachial artery). Seventy per cent of patients did not support their back during BP self-measurements. Additionally, the upper limb of $56 \%$ of patients was incorrectly placed. Other patient errors included: not being in a seated position (1\%), holding a conversation during measurements $(8 \%)$, failing to lay their fingers loosely (14\%), keeping their legs crossed $(20 \%)$, wearing clothing that compressed the shoulder region (22\%), and the incorrect placement of the cuff (27\%).

Figure 1 Number and types of errors made by patients during BP self-measurements.

\section{Type of errors and patient characteristics}

Older patients were most likely to lack back support $(p=0.042)$, while those with a higher BMI were more likely to incorrectly fasten the cuff $(p=0.014)$ and not relax their fingers during testing $(p=0.013)$. Associations between patient characteristics, sphygmomanometer type, and particular errors are presented in Table 1.

There was no observed dependence on errors regarding incorrect cuff position, pressure on the arm, or crossing the legs in relation to recorded patient characteristics.

\section{Number of errors and patient characteristics}

Patients inhabiting villages and towns with fewer than 50,000 inhabitants showed significantly more errors in recording their BP than patients living in larger cities $(\mathrm{p}=0.002)$. Patients using automatic and wrist sphygmomanometers showed fewer errors than those using aneroid sphygmomanometers $(\mathrm{p}=0.005)$.

\section{Association between the number of BP self-measurement errors and patient characteristics-multiple regression analysis}

Patients with longer HTN diagnoses made more errors than those diagnosed more recently $\left(r_{s}=0.201\right.$; $p=0.045)$.

Multiple regression analysis results are shown in Table 2 ( $p$ for model $=0.034 ; R^{2}=0.216$ ). Fewer errors were made by patients using upper $\operatorname{arm}(p=0.020)$ and wrist $(p=0.007)$ automatic sphygmomanometers compared to aneroid sphygmomanometers.

\section{Discussion}

Summary of main findings

Only $3 \%$ of the participants measured their BP without errors; $60 \%$ made three or more errors. The most common errors were using an incorrect pressure-gauge cuff, a lack of proper back support, and incorrect cuff placement on the upper limb. Patients living in cities and those using upper arm automatic, or wrist-arm devices made fewer errors than those living in villages or small towns and using aneroid gauges. Only onethird of patients received instructions on proper BP measurement from a healthcare professional; $22 \%$ did not receive any instructions.

\section{Strengths and limitations}

The methods used in this study regarding the recording and evaluation of BP self-measurement processes against standard measures are innovative.

However, this study presents some limitations. Firstly, the investigation was limited to one region of 


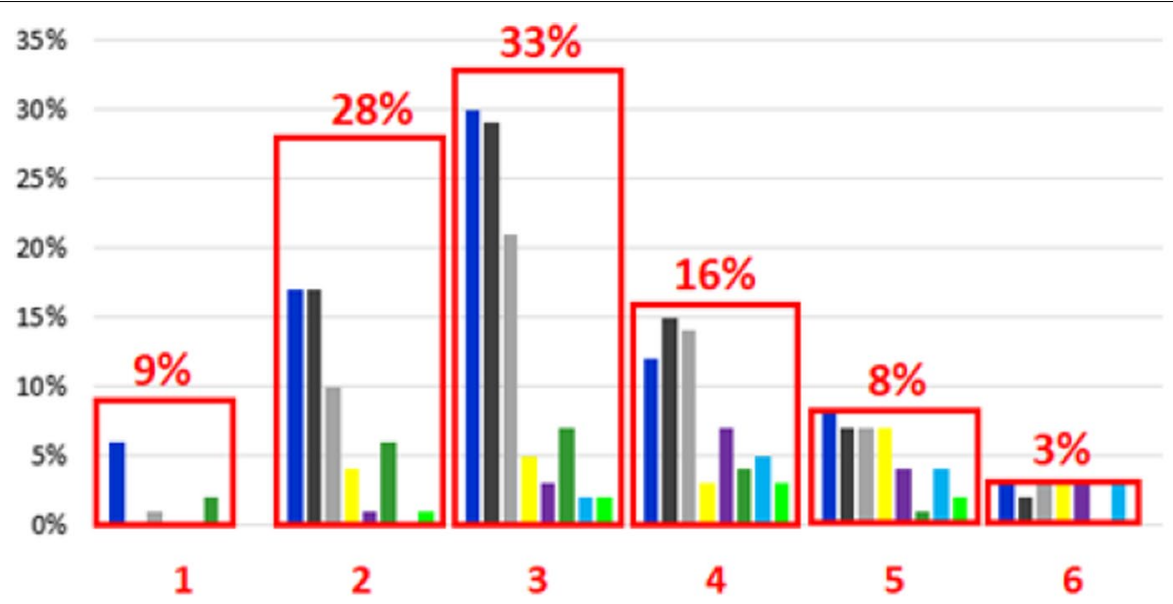

Incorrect pressure gauge cuff placement

No back support

Incorrect placement of the upper limb

Incorrect cuff fastening

compression of clothing on the frame

legs crossed

fingers not laid loosely

conversation during measurements

$\square$ Percentage of patients making a specific number of errors

Fig. 1 Number and types of errors made by patients during BP self-measurement

Poland, and the patients' sampling was not random. Accordingly, the study results cannot be generalized to the entire population of patients checking their BP at home. The enrolled participants measured their BP daily and, compared to the average patient, had a greater understanding of their overall health and were often more compliant with recommendations for therapy, ultimately increasing treatment success rates [15]. Secondly, patients measuring their BP in the clinic might perform a different routine than at home as they may feel stressed by researchers observing and recording the proceedings. Thus, one can expect that they measured their BP with a greater than normal level of attentiveness.

Also, it is necessary to add that all participants were requested to wait $5 \mathrm{~min}$ before they measured their BP. This procedure was a part of the protocol; however, it is uncertain whether patients would have paid sufficiently attention to this aspect of self-measurement at homes.
It should be further noted that this study was conducted in accordance with the 2010 ESH guidelines for HBPM [13] before the implementation of the $2021 \mathrm{ESH}$ guidelines [16]. However, the assessment of patient techniques for errors and the measurement techniques of the researchers were conducted in a manner also consistent with the new guidelines.

\section{Comparison with existing literature}

A study by Wagner et al. showed that a third of their patients were unable to accurately self-record their BP and that none of the collected measurements met technique recommendations in their entirety. As in this study, patients showed inaccurate recording techniques and a lack of understanding of how to check their BP [17].

$\mathrm{A}^{\prime}$ Court et al. assessed sphygmomanometer accuracy, finding digital devices to be as accurate as those with mercury and determining that aneroid monitors had higher failure rates [18]. In another study, the use of wrist 
Table 1 Associations between patients' characteristics, sphygmomanometer type and measurement errors

\begin{tabular}{|c|c|c|c|c|c|}
\hline \multirow[t]{2}{*}{ Characteristics of the patients } & \multicolumn{5}{|c|}{ Type of errors } \\
\hline & $\begin{array}{l}\text { Lack } \\
\text { of back } \\
\text { support }\end{array}$ & $\begin{array}{r}\text { Incorrect } \\
\text { upper limb position }\end{array}$ & $\begin{array}{l}\text { Incorrect cuff } \\
\text { fastening }\end{array}$ & $\begin{array}{l}\text { Fingers } \\
\text { relaxed }\end{array}$ & $\begin{array}{l}\text { during Talking } \\
\text { measurement }\end{array}$ \\
\hline Gender & & & $p=0.023$ & & \\
\hline Female & & & $36 \%$ & & \\
\hline Male & & & $12.9 \%$ & & \\
\hline Education & & & & & $p=0.037$ \\
\hline 1st-3rd EQF level & & & & & $4.88 \%$ \\
\hline 4th-6th EQF level & & & & & $2.94 \%$ \\
\hline 7th-8th EQF level & & & & & $20.00 \%$ \\
\hline Place of living & $p<0.001$ & & & & \\
\hline Village or town of less than 50,000 inhabitants & $38.71 \%$ & & & & \\
\hline City of more than 50,000 inhabitants & $84.06 \%$ & & & & \\
\hline Family history of hypertension & & & & $p=0.016$ & $p=0.020$ \\
\hline Yes & & & & $20.63 \%$ & $3.17 \%$ \\
\hline No & & & & $2.70 \%$ & $16.22 \%$ \\
\hline Type of the sphygmomanometer & & $p<0.001$ & & $p=0.001$ & $p=0.008$ \\
\hline Aneroid gauges & & $100 \%$ & & $27.27 \%$ & $27.27 \%$ \\
\hline Upper arm automatic & & $46.88 \%$ & & $10.94 \%$ & $3.13 \%$ \\
\hline Upper arm semi-automatic & & $14.29 \%$ & & $57.14 \%$ & $28.57 \%$ \\
\hline Wrist & & $77.78 \%$ & & $0 \%$ & $5.56 \%$ \\
\hline
\end{tabular}

Table 2 Multiple linear regression model: number of errors made during BP self-measurements associated with patient characteristics (reference group indicated in italics)

\begin{tabular}{|c|c|c|c|c|}
\hline \multicolumn{2}{|l|}{ Patient characteristics } & \multirow[t]{2}{*}{ Beta } & \multirow[t]{2}{*}{ b } & \multirow[t]{2}{*}{$p$} \\
\hline Variable & Comparison & & & \\
\hline \multicolumn{5}{|l|}{ Gender } \\
\hline Female & Male & -0.147 & -0.387 & 0.179 \\
\hline \multicolumn{5}{|l|}{ Education } \\
\hline \multirow[t]{2}{*}{ 1st-3rd EQF level } & 4th-6th EQF level & -0.106 & -0.287 & 0.331 \\
\hline & 7th-8th EQF level & -0.076 & -0.224 & 0.493 \\
\hline \multicolumn{5}{|l|}{ Place of living } \\
\hline $\begin{array}{l}\text { Village or town of less than } 50,000 \\
\text { inhabitants }\end{array}$ & City of more than 50,000 inhabitants & 0.188 & 0.521 & 0.093 \\
\hline \multicolumn{5}{|l|}{ Family history of hypertension } \\
\hline No & Yes & -0.056 & -0.148 & 0.606 \\
\hline \multicolumn{5}{|l|}{ Chronic comorbidities } \\
\hline No & Yes & -0.010 & -0.028 & 0.924 \\
\hline \multicolumn{5}{|l|}{ Type of sphygmomanometer } \\
\hline \multirow[t]{3}{*}{ Aneroid } & Upper arm automatic & -0.366 & -0.977 & 0.020 \\
\hline & Upper arm semi-automatic & -0.106 & -0.531 & 0.421 \\
\hline & Wrist & -0.414 & -1.381 & 0.007 \\
\hline Age & & -0.109 & -0.014 & 0.348 \\
\hline Time of hypertension diagnosis & & 0.157 & 0.024 & 0.153 \\
\hline $\mathrm{BMI}$ & & -0.045 & -0.012 & 0.700 \\
\hline
\end{tabular}


devices led to frequent detection of falsely elevated BP values [19].

Comparisons of BP measurements performed via "pragmatic" (measurements taken hastily with lower regard for the correct protocol to save time, such as in hectic medical settings) and standardised (as per protocol) methods were analysed in a study by Mlawanda et al. [19]. It was concluded that these two forms of measurements were different and should not be clinically interchanged. The study revealed that $16.7 \%$ of patients had their treatment options misclassified and that the mean BP was $143 / 90 \mathrm{mmHg}$ when measured pragmatically versus $133 / 87 \mathrm{mmHg}$ when measured in a standardised fashion.

González-López et al. assessed the knowledge of BP measurement procedures amongst Spanish medical and nursing students and revealed that only $51.8 \%$ of them knew how to measure their BP correctly [20]. More knowledge was demonstrated amongst nursing students over medical ones. However, the findings of another study conducted in Australia indicated that nurses had an inadequate knowledge base for performing BP measurements in a standardised manner and failed to prevent introduced error [21]. The above observations further highlight that even medical personnel experience difficulties with proper BP measurement techniques, putting into question their ability to educate patients on the subject.

Contrary to our findings, Nordmann et al. reported that patients could be trusted in reporting BP measurements accurately but that those with a low educational background should receive ambulatory measurements [22].

A study by $\mathrm{Li}$ et al. revealed that inappropriate cuff placement as an isolated error did not significantly affect the accuracy of BP measurements [23].

\section{Implications for research and clinical practice}

We interpret our results with caution. Nevertheless, the data suggest that most patients with HTN do not measure their BP correctly. This is not surprising as most subjects were never adequately counselled.

Although this study was a local one, there is no reason to expect that the greater population of hypertensive patients treated by family doctors in Poland perform their $\mathrm{BP}$ measurements more accurately than our subjects.

It was found that patients were less likely to make errors when measuring their BP while using automatic and wrist sphygmomanometers, which may be related to the differing levels of difficulty associated with using each device [24].

Residents of large cities made fewer mistakes than those living in small towns. It cannot be ruled out that they had greater access to specialist care, where they may have been trained to measure their BP.

Finally, it was determined that patients with a more extended history of HTN were more likely to make errors. These patients may have forgotten the correct measurement techniques they were taught previously and subsequently developed poor habits.

Should patients not measure their BP accurately, it may result in either a needed follow-up consultation not occurring or in increased strain on the healthcare system due to patients visiting their physician unnecessarily. In the latter case, there is an additional risk of patients being over-prescribed with excess or inappropriate medications, prompting further health risks.

Self-BP control has been proven to increase patient engagement and may improve adherence to HTN treatment [25-27]. However, our study has shown that patients make multiple errors as they self-measure their $\mathrm{BP}$, possibly negating the potential benefits of HBPM. The results suggest a strong need for family physicians to act and educate their patients on how to measure their BP at home correctly.

HBPM training by a physician or nurse should include verifying the patient's ability to measure their $B P$ independently [21]. Through standardised training offered by doctors or allied healthcare personnel, the accuracy of reported BP measurements by patients improve. Therefore, it is essential to underline that repeated careful instruction of correct BP measurement technique should be provided by medical staff to patients.

We believe that a larger scale study is needed to examine the influence of concomitant diseases and patient ages [28]. Future studies should also aim to determine which patient education methods are most effective and in which patient groups.

\section{Conclusions}

This study revealed that the majority of Polish hypertensive patients might make several errors when selfmeasuring their BP, significantly affecting their readings. Improperly measured BP in hypertensive patients can lead to poor disease control, increasing cardiovascular morbidity and mortality. The findings of this investigation reveal that self-administered BP measurements by patients are highly error prone. Therefore, healthcare professionals must do more to educate patients on proper $\mathrm{BP}$ measurement techniques specific to their device.

\footnotetext{
Abbreviations

BMI: Body mass index; BP: Blood pressure; ESH: European Society of Hypertension; EQF: European Qualifications Framework; GCP: Good clinical practice; HBPM: Home blood pressure monitoring; HTN: Hypertension; OBPM: Office blood pressure measurement.
} 


\section{Supplementary Information}

The online version contains supplementary material available at https://doi. org/10.1186/s12872-021-02351-5.

Additional file 1: Questionnaire used in the study.

Additional file 2: Detailed information on sample size calculation.

\section{Acknowledgements}

We would like to thank other medical students from the Students' Family Medicine Interest Group at JUMC in Kraków for helping to conduct this study, especially Natalia Augustyn and Katherine Kreciwilk. We are grateful to our patients for contributing their time and effort. Also, we wish to thank all the doctors from the practices from which the patients were recruited, especially Dr Krzysztof Studziński.

\section{Authors' contributions}

All authors mentioned contributed to the study. All authors read and approved the final manuscript. KN, MM, and AS gathered the data. AKK analysed the data. KN and MM reviewed the literature, analysed and interpreted the data, and drafted the manuscript. AW, MN, and AKK analysed and interpreted the data and revised the manuscript for important intellectual content. AW, KN, MN reviewed the literature and revised the manuscript for important intellectual content. AW and AS designed the questionnaire, designed the study, and revised the manuscript for important intellectual content. All authors read and approved the final manuscript.

\section{Funding}

None.

\section{Availability of data and materials}

Data from the full survey is available upon request.

\section{Declarations}

\section{Ethics approval and consent to participate}

Jagiellonian University Bioethics Committee nr. 122.6120.121.2015, received on June 25, 2015.

\section{Consent for publication}

Not applicable.

\section{Competing interests}

The authors declare that they have no competing interests.

\section{Author details}

'Department of Family Medicine, Jagiellonian University Medical College, Bocheńska 4, 31-061 Kraków, Poland. ${ }^{2}$ Department of Family Medicine, Students' Family Medicine Interest Group, Jagiellonian University Medical College, Kraków, Poland. ${ }^{3}$ Burns and Plastic Surgery Centre of Malopolska, Burns and Plastic Surgery Centre of Malopolska, Rydygier Memorial Hospital, Rydygier Memorial Hospital, Os. Zlotej Jesieni 1, 31-826 Kraków, Poland.

Received: 27 July 2021 Accepted: 18 October 2021

Published online: 12 November 2021

\section{References}

1. Ford ES. Trends in mortality from all causes and cardiovascular disease among hypertensive and nonhypertensive adults in the United States. Circulation. 2011;123(16):1737-44.

2. Chow CK, Teo KK, Rangarajan S, Islam S, Gupta R, Avezum A, et al. Prevalence, awareness, treatment, and control of hypertension in rural and urban communities in high-, middle-, and low-income countries. JAMA. 2013;04(9):959-68.

3. Zdrojewski T, Bandosz P, Rutkowski M, Gaciong Z, Grodzicki T, Wojtyniak B, et al. Rozpowszechnienie, wykrywanie i skuteczność leczenia nadciśnienia tętniczego w Polsce: wyniki badania NATPOL 2011 (The prevalence, detection, and effectiveness of the treatment of arterial hypertension in Poland: results of the NATPOL 2011 study). Nadciś Tętn. 2014;18:116-7.

4. Williams B, Mancia G, Spiering W, Agabiti Rosei E, Azizi M, Burnier M, et al. 2018 ESC/ESH Guidelines for the management of arterial hypertension: the task force for the management of arterial hypertension of the European Society of Cardiology (ESC) and the European Society of Hypertension (ESH). Eur Heart J. 2018;39(33):3021-104.

5. Lurbe E, Agabiti-Rosei E, Cruickshank JK, Dominiczak A, Erdine S, Hirth A, et al. 2016 European Society of Hypertension guidelines for the management of high blood pressure in children and adolescents. J Hypertens. 2016;34(10):1887-920.

6. Satoh M, Maeda T, Hoshide S, Ohkubo T. Is antihypertensive treatment based on home blood pressure recommended rather than that based on office blood pressure in adults with essential hypertension? (metaanalysis). Hypertens Res. 2019;5(6):807-16.

7. Mancia G, Bombelli M, Cuspidi C, Facchetti R, Grassi G. Cardiovascular risk associated with white-coat hypertension: pro side of the argument. Hypertension. 2017;70(4):668-75.

8. Ward AM, Takahashi O, Stevens R, Heneghan C. Home measurement of blood pressure and cardiovascular disease: systematic review and metaanalysis of prospective studies. J Hypertens. 2012;30(3):449-56.

9. McManus RJ, Mant J, Bray EP, Holder R, Jones MI, Greenfield S, et al. Telemonitoring and self-management in the control of hypertension (TASMINH2): a randomised controlled trial. Lancet. 2010;376(9736):163-72.

10. McManus RJ, Mant J, Haque MS, Bray EP, Bryan S, Greenfield SM, et al. Effect of self-monitoring and medication self-titration on systolic blood pressure in hypertensive patients at high risk of cardiovascular disease: the TASMIN-SR randomized clinical trial. JAMA. 2014;312(8):799-808.

11. Turner MJ, Baker AB, Kam PC. Effects of systematic errors in blood pressure measurements on the diagnosis of hypertension. Blood Press Monit. 2004;9(5):249-53.

12. Vischer AS, Burkard T. Principles of blood pressure measurement - current techniques, office vs ambulatory blood pressure measurement. Adv Exp Med Biol. 2017;956:85-96.

13. Parati G, Stergiou GS, Asmar R, de Leeuw P, Imai Y, Kario K, Lurbe E, Manolis A, Mengden T, O'Brien E, Ohkubo T, Padfield P, Palatini P, Pickering TG, Redon J, Revera M, Ruilope LM, Shennan A, Staessen JA, Tisler A, Waeber B, Zanchetti A, Mancia G. European Society of Hypertension Practice Guidelines for home blood pressure monitoring. J Hum Hypertens. 2010:24:779-85.

14. Department of Strategy, Qualifications, and Vocational Training of the Polish Ministry of Education: Coordination point for Polish and European qualifications framework. The Republic of Poland: The Polish Ministry of Education. 2017. https://prk.men.gov.pl/polska-rama-kwalifikacji-prk-ieuropejska-rama-kwalifikacji-erk/. Accessed 24 Apr 2020.

15. Warren RE, Marshall T, Padfield PL, Chrubasik S. Variability of office, 24-hour ambulatory, and self-monitored blood pressure measurements. Br J Gen Pract. 2010;60(578):675-80.

16. Parati G, Stergiou GS, Bilo G, Kollias A, Pengo M, Ochoa JE, Agarwal R, Asayama K, Asmar R, Burnier M, De La Sierra A, Giannattasio C, Gosse P, Head G, Hoshide S, Imai Y, Kario K, Li Y, Manios E, Mant J, McManus RJ, Mengden T, Mihailidou AS, Muntner P, Myers M, Niiranen T, Ntineri A, O'Brein E, Octavio JA, Ohkubo T, Omboni S, Padfield P, Palatini P, Pellegrin D, Postel Vinay N, Ramirez AJ, Sharman JE, Shennan A, Silva E, Topouchian J, Torlasco C, Wang JG, Weber MA, Whelton PK, White WB, Mancia G. Home blood pressure monitoring: methodology, clinical relevance and practical application: a 2021 position paper by the Working Group on Blood Pressure Monitoring and Cardiovascular Variability of the European Society of Hypertension. J Hypertens. 2021;39(9):1742-67.

17. Wagner S, Buus NH, Jespersen B, Ahrendt P, Bertelsen OW, Toftegaard TS. Measurement adherence in the blood pressure self-measurement room. Telemed J E-Health. 2013;19(11):826-33.

18. A'Court C, Stavens R, Sanders S, Ward A, McManus R, Heneghan C. Type and accuracy of sphygmomanometers in primary care: a cross-sectional observational study. Br J Gen Pract. 2011;61(590):e598-603.

19. Mlawanda G, Pather M, Govender S. An analysis of blood pressure measurement in a primary care hospital in Swaziland. Afr J Prim Health Care Fam Med. 2014:9(1):590. 
20. González-López JJ, Ramírez JG, García TR, Esteban SA, Alió del Barrio J, Rodríguez-Artalejo F. Knowledge of correct blood pressure measurement procedures among medical and nursing students. Rev Esp Cardiol. 2009;62(05):568-71.

21. Armstrong RS. Nurses' knowledge of error in blood pressure measurement technique. Int J Nurs Pract. 2002;8(3):118-26.

22. Nordmann A, Frach B, Walker T, Martina B, Battegay E. Reliability of patients measuring blood pressure at home: prospective observational study. BMJ. 1999:30(7218):1172.

23. LiY, Li F, Li Y, Cui X, Li J, Zhi H, Wang W, Sun Y, Cui W. Effect of cuff positioning on the accuracy of blood pressure measurement with automated electronic blood pressure monitors. J Clin Hypertens (Greenwich). 2020;22(7):1163-72

24. Casiglia E, Tikhonoff V, Albertini F, Palatini P. Poor reliability of wrist blood pressure self-measurement at home: a population-based study. Hypertension. 2016;68(4):896-903.

25. McManus RJ, Mant J, Haque MS, Bray EP, Bryan S, Greenfield SM, et al. Effect of self-monitoring and medication self-titration on systolic blood pressure in hypertensive patients at high risk of cardiovascular disease: the TASMIN-SR randomized clinical trial. JAMA. 2014:312(8):799-808.
26. McManus RJ, Mant J. Do differences in blood pressure between arms matter? Lancet. 2012;379(9819):872-3.

27. McManus RJ, Mant J, Franssen M, Nickless A, Schwartz C, Hodgkinson J, et al. Efficacy of self-monitored blood pressure, with or without telemonitoring, for titration of antihypertensive medication (TASMINH4): an unmasked randomised controlled trial. Lancet. 2018;391 (10124):949-59.

28. Parati G, Omboni S, Compare A, Grossi E, Callus E, Venco A, et al. Blood pressure control and treatment adherence in hypertensive patients with metabolic syndrome: protocol of a randomized controlled study based on home blood pressure telemonitoring vs. conventional management and assessment of psychological determinants of adherence (TELEBPMET Study). Trials. 2013;23:22.

\section{Publisher's Note}

Springer Nature remains neutral with regard to jurisdictional claims in published maps and institutional affiliations.
Ready to submit your research? Choose BMC and benefit from:

- fast, convenient online submission

- thorough peer review by experienced researchers in your field

- rapid publication on acceptance

- support for research data, including large and complex data types

- gold Open Access which fosters wider collaboration and increased citations

- maximum visibility for your research: over $100 \mathrm{M}$ website views per year

At BMC, research is always in progress.

Learn more biomedcentral.com/submissions 